\title{
EPITHELIAL REGENERATION AND PRESERVATION OF TRACHEAL CARTILAGE AFTER TRACHEAL REPLACEMENT WITH CRYOPRESERVED ALLOGRAFT IN THE RAT
}

Takashi Tojo, MD

Soichiro Kitamura, MD*

Satoshi Gojo, MD

Keiji Kushibe, MD

Kunimoto Nezu, MD

Shigeki Taniguchi, MD
Objective: We investigated the origin of the epithelium in transplanted cryopreserved tracheal allografts in rats and tried to clarify the mechanism by which immunogenicity is reduced in this procedure. Methods: Tracheal transplantation was performed with PVG rats (allele at the RT1 locus: c) used as donors and ACI rats (allele at the RT1 locus: a) as recipients. After resection of a 5-ring segment of the cervical trachea of an ACI rat, the trachea was reconstructed with the cryopreserved tracheal segment of a PVG rat $(n=6)$. No immunosuppressive agents or steroids were given. Histologic changes were determined and immunohistochemical staining was performed to investigate major histocompatibility complex class I antigens of the transplanted tracheal segment. Results: Two months after tracheal transplantation, 6 surviving ACI rats were killed. Histologically, the epithelium and tracheal cartilage of the transplanted cryopreserved segment displayed normal structure. Immunohistochemical staining showed that the major histocompatibility complex class I antigen of the ACI rat was expressed in the epithelium of the transplanted segment and that the class I antigen of the PVG rat was expressed in the cartilage of the transplanted segment. Conclusions: After transplantation of the cryopreserved trachea, the epithelium of the transplanted cryopreserved segment originated from the recipient epithelium whereas the cartilage retained the structure of the donor trachea. We hypothesize that transplantation of a cryopreserved trachea leads to the growth of the recipient's epithelium over the donor trachea, thereby reducing the antigenicity of the transplant. (J Thorac Cardiovasc Surg 1998;116:624-7)
A compared with other organs, the trachea is generally believed to have a weak rejection reaction to grafts. ${ }^{1}$ However, the tracheal allograft may be rejected if immunosuppression is not administered. ${ }^{2-5}$ Studies of antigenicity have shown that the tracheal epithelium may play an important role in graft rejection., ${ }^{6,7} \mathrm{Re}-$ ducing the antigenicity of the allograft itself is an excellent method for controlling the immunologic reaction. For this purpose, cryopreservation techniques were applied and were fairly effective in maintaining viability

From the Department of Surgery, Nara Medical University, Nara, Japan.

Received for publication May 29, 1997; revisions requested Sept 2, 1997; revisions received Feb 13, 1998; accepted for publication April 24, 1998.

Address for reprints: Takashi Tojo, MD, Department of Surgery III, Nara Medical University, 840, Shijo-cho, Kashihara, Nara, 634, Japan.

*Presently: National Cardiovascular Center.

Copyright $\odot 1998$ by Mosby, Inc.

$0022-5223 / 98 \$ 5.00+0 \quad \mathbf{1 2 / 1 / 9 1 3 7 4}$ and structural integrity and reducing the immunologic response. ${ }^{8-10}$ Previous experiments in dogs showed that after implantation of cryopreserved tracheal allografts, the trachea maintained its structure and no lymphocytic infiltration developed in or under the epithelial layer. ${ }^{11-14}$ However, the reason for this phenomenon is still unclear. In this study, we investigated the origin of the epithelium in transplanted cryopreserved tracheal allografts and tried to clarify the mechanism by which immunogenicity in cryopreserved tracheal allografts is reduced.

\section{Materials and methods}

Animal strains. Experiments were performed on the following inbred rat strains: (1) PVG rat: RT1 haplotype is c; (2) ACI rat: RT1 haplotype is a.

The following monoclonal antibodies were used for recognizing a major histocompatibility complex (MHC) class I determinant: (1) anti-rat RT1.A recognizes an MHC class I determinant, haplotypes c and n (Cedarlane Laboratories, Ltd, Hornby, Ontario, Canada); (2) anti-rat RT1.A recognizes the rat MHC class I antigen, haplotypes a and b, and does not 

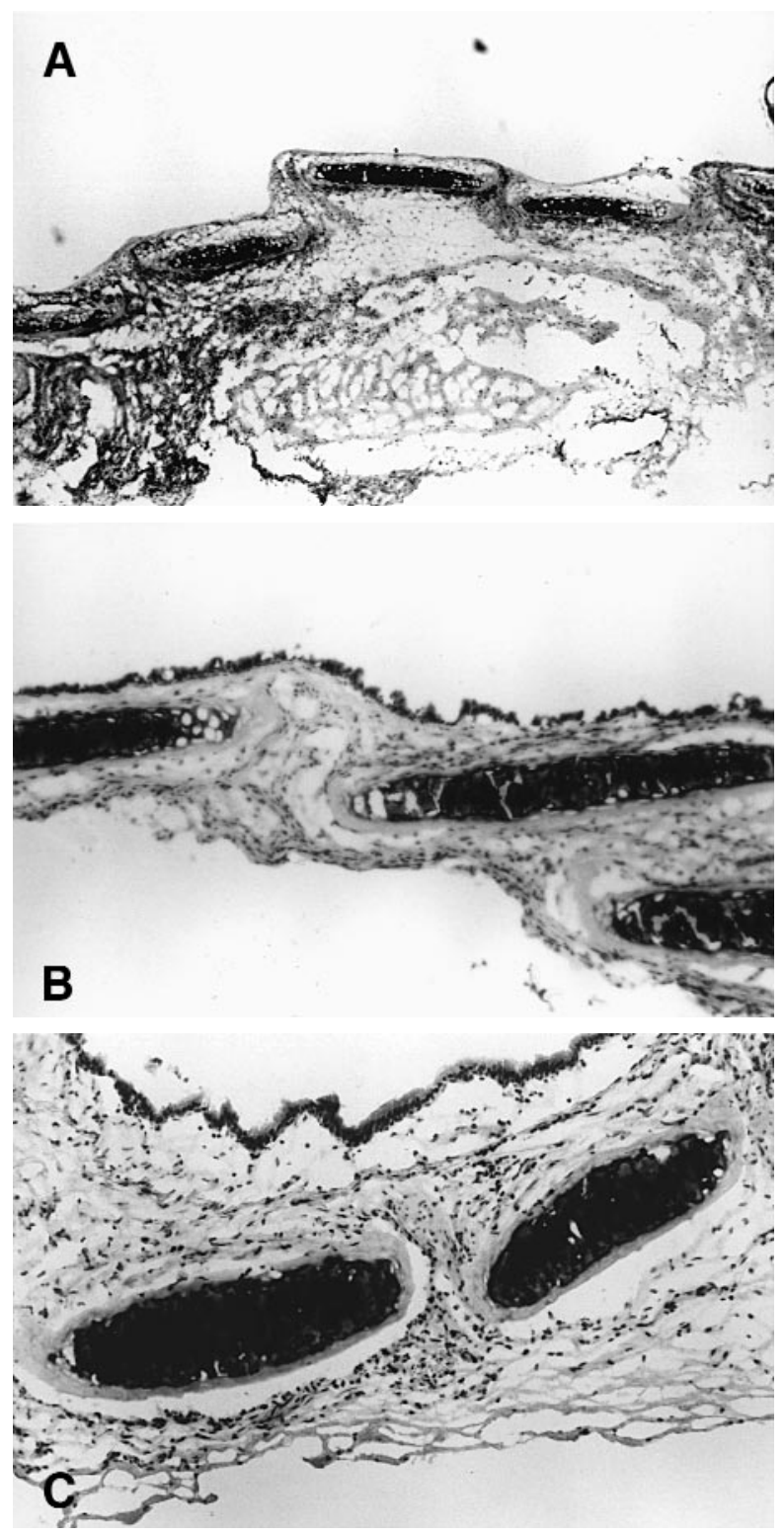

Fig 1. Histologic investigations of the cryopreserved tracheal segment was performed at 3 days (A), 1 week (B), and 2 months (C) after transplantation $(\times 40)$. Three days after transplantation, the epithelium of the transplanted segment had disappeared, and in 1 week it grew sparsely. Two months after transplantation, the epithelium and tracheal cartilage of the transplanted cryopreserved segment displayed normal histologic structural integrity.

react with haplotypes c, 1, and u (PharMingen, San Diego, Calif).

Tracheal transplantation. Tracheal transplantation was performed with PVG rats used as donors and ACI rats as recipients. Tracheal allografts for cryopreservation were prepared from healthy PVG rats. The freezing medium was TC199 solution with a final concentration of $5 \%$ HEPES buffer
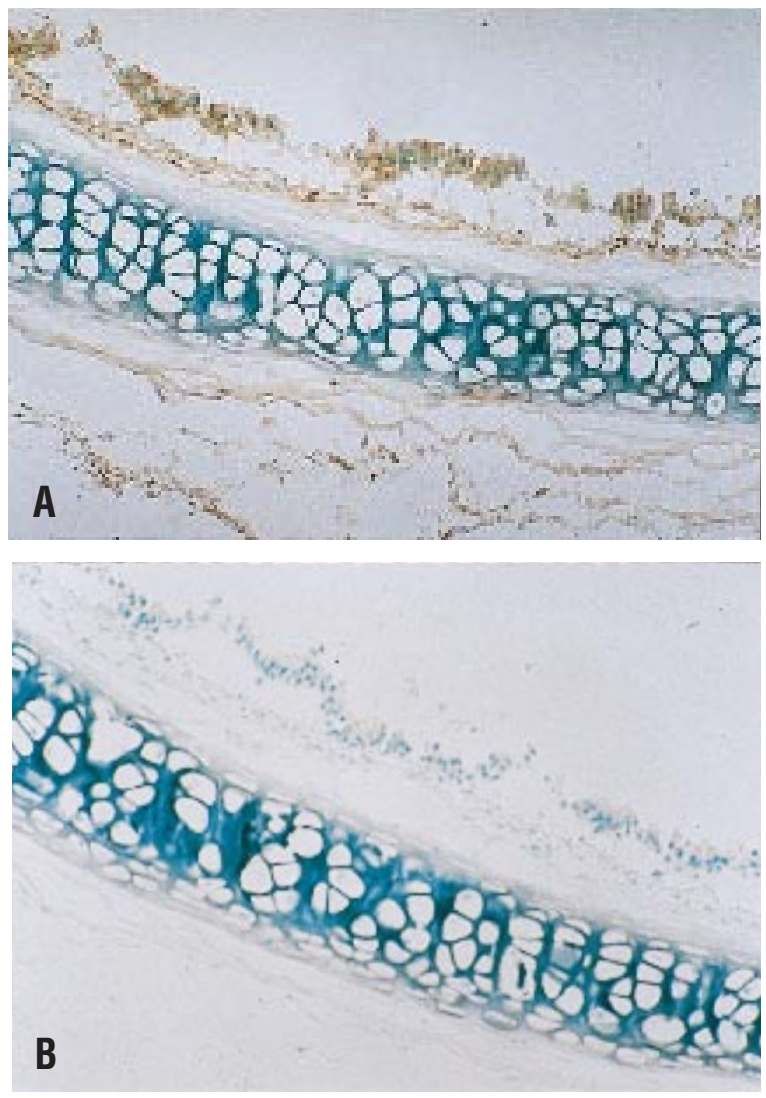

Fig 2. Immunohistochemical staining of a cryopreserved tracheal segment of PVG rat before transplantation was performed. A, Tracheal segment stained with anti-rat RT1. $\mathrm{A}^{\mathrm{c} \text { and } \mathrm{n}}$ (anti-PVG rat). B, Segment stained with anti-rat RT1.A $\mathrm{A}^{\mathrm{a} \text { and } \mathrm{b}}$ (anti-ACI rat). MHC class I antigen of the PVG rat (c haplotype) was expressed in the epithelium and the cartilage stained by anti-rat RT1.A $\mathrm{c}^{\mathrm{c} \text { and } \mathrm{n}}$.

and $10 \%$ dimethyl sulfoxide. A sterile plastic bag containing the specimen was filled with the freezing medium, sealed, and frozen at a rate of $-1^{\circ} \mathrm{C} / \mathrm{min}$ to $-80^{\circ} \mathrm{C}$ by a programmed freezer. The bag was then stored in liquid nitrogen (at $-196^{\circ} \mathrm{C}$ ) for 1 to 2 months. At implantation, the specimen was thawed by placing the bag in a $40^{\circ} \mathrm{C}$ water bath and rinsing out the freezing medium.

After being euthanized with an intra-abdominal injection of atropine sulfate $(1 \mathrm{mg} / \mathrm{kg})$ and pentobarbital sodium (10 $\mathrm{mg} / \mathrm{kg}$ ), an ACI rat (weighing $263.3 \pm 34.3 \mathrm{~g}$ ) was placed in the supine position. After a skin incision and a median split in the cervicolongitudinal muscle were made, the cervical trachea was exposed. A 5-ring segment of the cervical trachea was resected, and the trachea was then reconstructed with the cryopreserved tracheal segment from a PVG rat. Anastomoses were performed with 3 or 4 interrupted sutures of 7-0 polypropylene. During the proximal and distal anastomoses, ventilation was maintained by spontaneous breathing through a 16-gauge orotracheal tube. No immunosuppressive agents or steroids were given to the rats. 

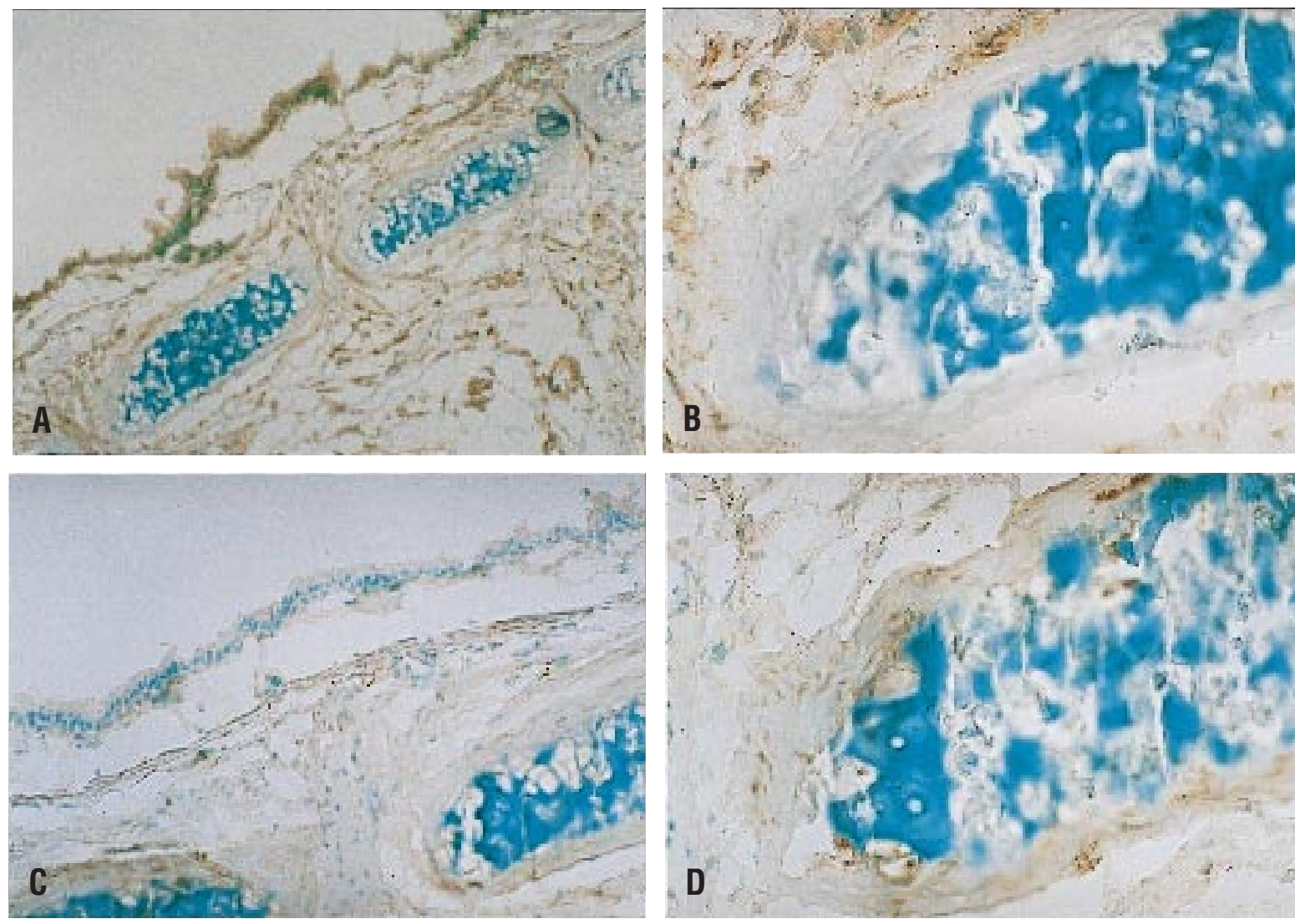

Fig. 3. Immunohistochemical staining of the transplanted cryopreserved tracheal segment of PVG rats at 2 months after transplantation. A and B, Tracheal segment stained with anti-rat RT1.A ${ }^{\mathrm{a} \text { and } \mathrm{b}}$ (anti-ACI rat). C and D, Tracheal segment stained with anti-rat RT1. $\mathrm{A}^{\mathrm{c} \text { and } \mathrm{n}}$ (anti-PVG rat). MHC class I antigen of the ACI rat was expressed in the epithelium of the transplanted segment, and MHC class I antigen of the PVG rat was expressed in the cartilage of the transplanted segment.

Two months after tracheal transplantation, the animals were killed and the tracheal grafts were examined grossly and microscopically. Immunohistochemical staining was performed with the anti-rat RT1.A monoclonal antibody that recognizes an MHC class I determinant.

All animals were treated in compliance with the "Principles of Laboratory Animal Care" formulated by the National Society for Medical Research, the "Guide for the Care and Use of Laboratory Animals" prepared by the Institutes of Laboratory Animal Resources and published by the National Institutes of Health (NIH Publication No. 86-23, revised 1985), and the "Guidelines for Animal Experimentation" published by the Japanese Association for Laboratory Animal Science (Exp. Anim. 36(3), 285-288, 1987).

\section{Results}

Twelve tracheal transplants were performed and 2 ACI rats died within a week after the operation. Of the 10 ACI rats that survived, 2 were killed and examined 3 days after the operation, 2 rats 1 week after the oper- ation, and 6 rats 2 months after the operation. Histologic changes were determined and immunohistochemical staining was performed to investigate $\mathrm{MHC}$ class I antigens of the transplanted tracheal segment.

Histologic investigation. Three days after transplantation, the epithelium of the transplanted segment had disappeared, and in 1 week it grew sparsely. Two months after the operation, the epithelium and tracheal cartilage of the transplanted cryopreserved segment displayed normal histologic structural integrity. The epithelium had pseudostratified columnar features with ciliated, mucous, and basal cells in these segments; the cartilaginous architecture was not disrupted. No lymphocytic infiltration was detected in or under the epithelial layer in these segments (Fig 1).

Immunohistochemical staining. MHC class I antigens were expressed in the cryopreserved tracheal segments of PVG rats with the use of anti-rat RT1. $\mathrm{A}^{\mathrm{c} \text { and }} \mathrm{n}$ (Fig 2). After transplantation of cryopreserved tracheal 
segments of PVG rats, MHC class I antigen of the ACI rat was expressed in the epithelium of the transplanted segment, and MHC class I antigen of the PVG rat was expressed in the cartilage of the 6 transplanted segments (Fig 3). In transplantation of cryopreserved trachea, the epithelium of the transplanted cryopreserved segment originated from the recipient epithelium, and the cartilage retained the structure of the donor trachea.

\section{Discussion}

Tracheal allografts may be rejected without immunosuppression, and the epithelium of the trachea may play an important role in such rejection..$^{2-4}$ In controlling immunologic reactions, use of immunosuppressants certainly attenuates graft rejection, but these agents can also increase airway infection after tracheal replacement. Therefore reducing the antigenicity of the allograft itself is a preferred alternative. Cryopreservation techniques are favored for maintaining viability and structural integrity and reducing the immunologic response of several tissues. ${ }^{8-10}$ This method has been applied to experimental tracheal allotransplantation, and immunosuppressant-free tracheal allotransplantation has been carried out successfully. ${ }^{11-14}$ In these experiments, eliminating the epithelium was hypothesized to have played an important role in the success of cryopreserved tracheal allotransplantation. Inasmuch as the epithelium of the transplanted segment was well monitored after transplantation, it was unclear whether the epithelium that grew belonged to the donor or the recipient. In other words, was the donor's epithelium (in which antigenicity was reduced by cryopreservation) growing, or did the recipient's epithelium develop after the elimination of the donor's epithelium? We investigated the origin of the epithelium of the transplanted cryopreserved segment and proposed a mechanism by which antigenicity is reduced in cryopreserved tracheal allografts.

In our experiment, the tracheal epithelium was preserved after freezing and thawing of the donor trachea. However, 2 months after replacement of the donor trachea, the epithelium of the donor had disappeared and the epithelium of the recipient had developed over the transplanted donor trachea. After cryopreservation, the structure of the tracheal epithelium is preserved; however, after transplantation, the epithelium of the donor is replaced by the epithelium of the recipient on the donor trachea. Therefore we conclude that antigenicity is reduced by the growth of the recipient's epithelium over that of the donor.

\section{REFERENCES}

1. Rose KG, Sesterhenn K, Wustrow F. Tracheal allotransplantation in man [letter]. Lancet 1979;1:433.

2. Alonso WA, Bridger GP, Bordley JE. Tracheal transplantation in dogs. Laryngoscope 1972;82:204-9.

3. Beigel A, Muller-Ruchholtz W. Tracheal transplantation. I. The immunogenic effect of rat tracheal transplants. Arch Otorhinolaryngol 1984;240:185-92.

4. Beigel A. Muller-Ruchholtz W. Tracheal transplantation. II. Influence of genetic difference and degree of sensitization on reaction to the tracheal transplants. Arch Otorhinolaryngol 1984;240:217-25.

5. Farrington WT, Hung WC, Binns PM. Experimental tracheal transplantation. J Laryngol 1977;91:101-10.

6. Bujia J, Wilmes E, Hammer C, Kastenbauer E. Tracheal transplantation: demonstration of HLA class II subregion gene products on human trachea. Acta Otolaryngol (Stockh) 1990;110:14954.

7. Kalb TH, Chuang MT, Marom Z, Mayer L. Evidence for accessory cell function by class II MHC antigen-expressing airway epithelial cells. Am J Respir Cell Mol Biol 1991;4:320-9.

8. Boren $\mathrm{CH}$, Roon AJ, Moore WS. Maintenance of viable arterial allografts by cryopreservation. Surgery 1977;83:382-91.

9. Kawabe N, Yoshinao M. Cryopreservation of cartilage. Int Orthop 1990;14:231-5.

10. Kirklin JK, Smith D, Novick W, Naftel DC, Kirklin JW, Pacifico $\mathrm{AD}$, et al. Long-term function of cryopreserved aortic homografts; a ten-year study. J Thorac Cardiovasc Surg 1993;106:154-66.

11. Messineo A, Filler RM, Bahoric A, Smith CR. Repair of tracheal defects with cryopreserved cartilaginous allografts. J Pediatr Surg 1992;27:1131-5.

12. Tojo T, Sawabata N, Nezu K, Kawachi K, Kitamura S. Tracheal allogenic immunoresponse is reduced by cryopreservation: canine experiment. Transplant Proc 1996;28:1814-5.

13. Yokomise H, Inui K, Wada H, Ueda M, Hitomi S. Long-term cryopreservation can prevent rejection of canine tracheal allografts with preservation of graft viability. J Thorac Cardiovasc Surg 1996;111:930-4.

14. Inutsuka K, Kawahara K, Takachi T, Okabayashi K, Shiraishi T, Shirakusa T. Reconstruction of trachea and carina with immediate or cryopreserved allografts in dogs. Ann Thorac Surg 1996;62:1480-4. 